\title{
Anti-thyroglobulin antibodies do not significantly increase the risk of finding iodine avid metastases on post-radioactive iodine ablation scan in low-risk thyroid cancer patients
}

\author{
F. Nabhan ${ }^{1}$, K. Porter ${ }^{2}$, L. Senter ${ }^{3}$, M. D. Ringel ${ }^{1}$ \\ ${ }^{1}$ Division of Endocrinology, Diabetes, and Metabolism, The Ohio State University Medical Center, \\ The Ohio State University and Arthur G. James Cancer Center, 575 McCampbell Hall, 1581 Dodd \\ Dr, Columbus, OH 43210, USA \\ ${ }^{2}$ Center for Biostatistics, Department of Biomedical Informatics, The Ohio State University College \\ of Medicine, Columbus, $\mathrm{OH}$ 43235, USA \\ ${ }^{3}$ Division of Human Genetics, The Ohio State University and Arthur G. James Cancer Center, \\ Columbus, $\mathrm{OH} 43235$, USA
}

\begin{abstract}
Objective-Post-operative thyroglobulin ( $\mathrm{Tg}$ ) levels can predict the likelihood of residual cancer, including distant metastases, thereby influencing postsurgical treatment strategies even in patients with low-risk disease. Circulating anti-thyroglobulin antibodies (anti-Tg Abs) interfere with $\mathrm{Tg}$ measurement preventing this clinical use. It is not known if the presence of anti-Tg Abs predicts metastatic disease on post-therapy scan in patients with low-risk disease or if they should influence the use or dose of I-131 therapy. In the present study, we compare post-therapy scans in low-risk patients with and without anti-Tg Abs.
\end{abstract}

Methods-This is a single-institution retrospective study. The study population (Group A) included all patients with low-risk differentiated thyroid cancer (DTC) who underwent total thyroidectomy and RAI between 1/1/2006 to 9/11/2015 with intrathyroidal T1-T2, Nx, N0 or N1a ( 5 nodes all measuring, when reported, $<2 \mathrm{~mm}$ ) that had anti-thyroglobulin antibodies. Patients were excluded if they had known distant metastases and/or extensive vascular invasion. A second group of patients (Group B) treated during the same period but without anti-Tg antibodies was selected to match group A by propensity core matching with a logistic regression model.

Results-Each group included 37 patients. In group A: Median age was 40 years, $86 \%$ female and $76 \%$ PTC. Median tumor size was $2 \mathrm{~cm}(0.2-3.8), 32 \%$ had multifocal disease, $16 \%$ were N1a and $4 \%$ had vascular invasion. Parameters in group B were not statistically different from Group A, as expected based on the selection criteria, except being less likely to have Hashimoto's thyroiditis on pathology $(p<0.001)$. Post-therapy scan results were compared by Chi-square test

F. Nabhan, fadi.nabhan@osumc.edu.

Conflict of interest The authors have no conflict of interest.

Ethical approval This study was approved by the Ohio State University Institutional Review Board.

Informed consent Informed consent was obtained from all individual participants included in the study. 
with $86 \%$ negative post therapy scan frequency in group A and $92 \%$ in group B without evidence of a difference $(p=0.45)$.

Conclusion-In patients with low-risk DTC, anti-Tg Abs did not significantly predict metastatic disease on post-therapy scan. If confirmed, these data suggest that the presence of anti-Tg Abs alone should not influence initial therapy in patients with low-risk DTC.

\section{Keywords}

Thyroid cancer; Anti-thyroglobulin antibodies; Radioactive iodine ablation

\section{Introduction}

The decision to prescribe radioactive iodine (RAI) after total thyroidectomy is based on the risk stratification according to the pathological findings and the postoperative assessment of disease status including serum thyroglobulin ( $\mathrm{Tg}$ ) level [1]. Detecting radioactive iodine avid metastases on post-therapy (Post-Tx) scan is one potential benefit of treating with RAI. The level of Tg measured after total thyroidectomy can predict the likelihood of radioactive iodine avid metastases [2,3], and therefore can help select the group of patients in whom RAI may help detecting unknown iodine avid disease and thus help guide the decision of whether to prescribe RAI. This role of Tg in clinical decision-making is particularly important in patients with low risk thyroid cancers in whom RAI administration may not be indicated based on pathology information alone [1].

Anti-Thyroglobulin antibodies (anti-Tg Abs) interfere with the measurement of $\mathrm{Tg}[4,5]$ making Tg not a useful test in these patients. Because many thyroid cancer studies exclude patients with positive anti-Tg Abs there is a relative paucity of data in this population to make definitive management recommendations, including whether their presence should influence decisions regarding RAI therapy. The NCCN guidelines, for example, use Tg level cutoff as part of the overall approach to the decision-making regarding RAI and recommend "consideration" of RAI if the patient has positive anti-Tg Abs [6]. Such recommendations suggest that an important knowledge gap exists in management of these individuals.

In this study, we evaluate whether the presence of anti-Tg antibodies is associated with specific aspect of the rationale for using RAI, which is to detect or exclude radioactive iodine avid metastatic disease on Post-Tx scans. Our aim is to compare the Post-Tx scans in patients with and without anti-Tg Abs after total thyroidectomy in patients with pathological findings indicating low-risk disease.

\section{Materials and methods}

\section{Patients}

This is a retrospective cohort study conducted at The Ohio State University (OSU)Wexner Medical Center and Arthur G. James Cancer Hospital and Solove Research Institute, the Ohio State Comprehensive Cancer Center, and was approved by the Ohio State University Institutional Review Board. Patients were identified through a query of the Ohio State University Endocrine Neoplasia Repository database. This repository is open to all thyroid 
cancer patients seen at any time during the course of treatment and follow-up for their disease. We evaluated records of consecutive patients who were treated with RAI from 1/1/2006 until 9/11/2015. We included only patients with histological well-differentiated follicular cell-derived thyroid cancer (DTC) with T1 or T2 tumor size that had either no or minimal ( $\leq$ nodes and all, when reported, measured $<2 \mathrm{~mm}$ ) node involvement who underwent total thyroidectomy (with or without central neck dissection) and received radioactive iodine therapy with available post therapy scan reports. We excluded those with known distant metastases, high-risk cell variants, extra thyroidal extension, extensive nodal metastases (see above), and extensive vascular invasion (if it was reported in the pathology report or if the number of vascular space invasion exceeded 4). We also excluded patients with lateral neck lymph node metastases as lateral neck dissection will be performed only if macroscopic disease is found pre-operatively. The patients were then divided into two groups: group A which included all patients who met the above criteria and were positive for anti-Tg Abs at the time of radioactive iodine ablation and group B which was selected to match group A (see statistical analysis below) from those patients who were low risk, treated with RAI but who were negative for anti-Tg Abs at the time of RAI. Patients were included if they had their RAI whether at our institutions or other institutions as long as there was a post therapy scan result available and $\mathrm{Tg}$ and anti-Tg Abs were measured. RAI was administered following either recombinant TSH injections or thyroid hormone withdrawal (THW) achieving a TSH $>30 \mathrm{mIU} / \mathrm{L}$.

\section{Tg and anti-Tg Abs assays}

Over the course of time of the data acquisition, there were changes in the $\mathrm{Tg}$ and $\mathrm{Tg}$ antibody assays utilized. Depending on the time of the thyroid cancer diagnosis, $\mathrm{Tg}$ was measured by two different immunometric assays (referred to in paper as Tg-IMA, whether by Siemens Immulite or Beckman Coulter assays). The two assays used for Tg measurement were the following: (1) Immulite 2000 XPi Thyroglobulin assay (Siemens Inc., Deerfield, IL; catalog no. PIL2KTY, Tg-I in the manuscript) which has an analytical sensitivity (limitof-detection) defined as $<0.2 \mathrm{ng} / \mathrm{mL}$ and a functional sensitivity (limit-of-quantitation) defined by a CV $<20 \%$ of $0.9 \mathrm{ng} / \mathrm{mL}$. (2) Beckman Access Tg (Beckman Coulter, Tg-B in manuscript) was performed at Mayo Clinic Laboratories (Rochester, MN). The functional sensitivity was at $0.1 \mathrm{ng} / \mathrm{ml}$. Anti Tg antibodies were detected using three assay systems during the course of time of the study: (1) Immulite 2000 XPi anti-Thyroglobulin Antibody assay (Siemens Inc., Deerfield IL; catalog no. L2KTG2; anti-Tg Abs-I in manuscript) which is a two-step chemilu-minescent immunoassay for quantitation of antibodies against $\mathrm{Tg}$. The manufacturer analytical functional sensitivity is listed as $20 \mathrm{IU} / \mathrm{mL}$ or (2) Roche assay (antiTg Abs-R) performed at Mayo Medical Laboratories (Rochester, MN) with functional sensitivity of $20 \mathrm{IU} / \mathrm{ml}$ and (3) Access Thyroglobulin Antibody II assay (Beckman Coulter, Anti Tg Abs-B.). This was also performed at Mayo Medical Laboratories (Rochester, MN). The reported functional sensitivity was $1.8 \mathrm{IU} / \mathrm{ml}$. For this analysis, a detectable level of anti-Tg Abs according to the functional sensitivity (limit of quantification) on any one of the assays was reported as a positive result. 


\section{Statistical analysis}

Patients were divided into group A with positive anti-Tg Abs and group B with negative anti-Tg Abs. Group A included all patients who were of low risk, received RAI and were positive for anti-Tg Abs at the time of RAI. From the rest of low risk patients who received RAI and were negative for anti-Tg Abs, we selected patients (Group B) to match group A using propensity score matching with logistic regression model including age, gender, histology, N stage, and anti-Tg Abs assay. Post-therapy scans between the two groups were compared by Chi-square test.

\section{Results}

\section{Baseline characteristics}

The study included 37 patients in each group. Table 1 describes the characteristics of each group. Median age in group A was 40 years (range of 18-67), 86\% were female and 76\% were Papillary Thyroid Cancer (PTC) with the rest being Follicular Thyroid Cancer (FTC) and Hurthle Thyroid Cancer (HTC). Median size was $2 \mathrm{CM}$ with range of $0.2-3.8 \mathrm{~cm}$ where $49 \%$ were $\mathrm{T} 1$ and $51 \%$ were T2. Thirty-two percent (32\%) had multifocal disease. Sixteen percent (16\%) were N1a with 54\% being at N0 and 30\% at Nx. Fourteen percent (14\%) had minimal vascular invasion Table 1. Parameters in group B were not statistically different from group A except for the presence of Hashimoto's thyroiditis, which was more common in group A at 83 versus $27 \%$ in group B $(p<0.001)$. In group B and depending on available data on unstimulated versus stimulated $\mathrm{Tg}$ levels, mean levels of unstimulated $\mathrm{Tg}$ was 1.1 $\mathrm{ng} / \mathrm{ml}(\mathrm{SD}=1)$ and stimulated $\mathrm{Tg}$ was $15 \mathrm{ng} / \mathrm{ml}(\mathrm{SD}=16.4)$.

\section{Post-therapy scan results}

The median dose of RAI in the study group (A) was $50 \mathrm{mCi}$ (range of 30-151) compared to a median of $50 \mathrm{mCi}(30-150)$ in the matched group (B). Post therapy scan results were compared by Chi-square test with $86 \%$ negative post therapy scan frequency in group A and $92 \%$ in group B without evidence of a difference $(p=0.45$; Table 2$)$.

\section{Most recent follow-up status}

Median follow-up was at a median of 37 months in the study group and 35 months in the matched group. According to ATA classification, (1), in group A, 38\% were free of disease, $51 \%$ were indeterminate (19 patients, 16 of whom were considered indeterminate due to detectable anti-Tg Abs) and $11 \%$ unknown. None had biochemical incomplete response or structural disease. In the matched group (group B) with negative anti-Tg Abs, 76\% where free of disease, $19 \%$ were indeterminate, $3 \%$ were biochemically incomplete and $3 \%$ had structural disease. Since the assay for anti-Tg Abs was different at the final follow-up from the assay used at the time of RAI in the majority of patients, we could not report trend of anti-Tg Abs from time of RAI to final follow-up (Table 3).

\section{Discussion}

One of the rationales for administration of RAI is to assess presence of metastatic disease in post-tx scans in patients felt to be at risk for metastatic disease, to complete staging of the 
tumor at diagnosis. One of the features that helps define that risk is the post-thyroidectomy Tg level $[2,3]$. Because Tg levels in patients with positive anti-Tg Abs are unreliable and often underestimate disease burden, it cannot be used in that circumstance to help with treatment planning $[4,5]$. It is not conclusively known if the presence of anti-Tg antibodies after surgery predicts a higher or lower likelihood of distant metastases on post-therapy scan in patients with thyroid cancer. In this study, we evaluated the post-tx scan results of patients with low risk disease after total thyroidectomy that have positive anti-Tg Abs and compared that to a matched group with a similar risk but negative for anti-Tg Abs.

The key finding in this study is that a case-matched population of patients with low risk thyroid cancer; the likelihood of detecting iodine avid metastases was the same in patients with or without circulating anti-Tg Abs. Both groups indeed had a low frequency of iodine avid metastases on the post-tx scan, which is in agreement with prior reports in low risk patients $[7,8]$. The second key finding is that both groups had similar numbers of patients who developed structural disease during follow-up.

A number of studies have evaluated the clinical course of thyroid cancer patients with anti$\mathrm{Tg}$ Abs in comparison to individuals without Tg antibodies. The results have been mixed. Some studies reported that patients with positive anti-Tg Abs have a more aggressive clinical course $[9,10]$ or have evidence of more metabolically active disease on positron emission tomography (PET) [11] while others did not demonstrate that [12]. These results make clinical recommendations challenging. For example, Durante et al. [10] compared the course of patients with and without anti-Tg Abs and found that the anti-Tg Abs positive patients had a higher likelihood to present with high-risk disease than the anti-Tg Abs negative patients. In addition, individuals with anti-Tg antibodies were more likely to have recurrent or persistent disease during follow-up. In the present study, no such difference was identified; however, there are important distinctions between the two study populations: In the present study, all patients were low risk and all received RAI while in Durante et al. [10] the patients included were at variable risk and some did not receive RAI. The number of patients in our study was smaller than that in Durante et al. [10], which also may contribute to the absence of a significant difference being identified. Another difference is that the low risk disease in the present study was defined using criteria that were closer to the most updated ATA guidelines [1] including acceptance of low volume central cervical nodal metastases and minimal vascular invasion. It is noted, however, that even with the difference in risk of recurrent/persistent disease in our study as compared to Durnate et al. [10], both studies showed similarities in the rate of finding distant metastases on initial post-treatment scans between the positive and negative anti-Tg Abs patients.

While the presence of iodine avid metastases was not statistically significant between the two groups in the present study, it is recognized that the number of patients is small due in part to the strict exclusion criteria. In addition, since all iodine avid metastases found in group A were cervical nodes, many may have been discovered with other imaging tests such as ultrasound, without the need to administer RAI. Many studies reported that lack of benefits of RAI treatment in low-risk patients without positive anti-Tg Abs based on the absence of a reduction of recurrence risk or improved disease-specific survival [13-18]. Our data support this less aggressive approach, even in the presence of anti-Tg Abs. 
The present retrospective study cannot address the potential benefit of higher rate of disappearance of anti-Tg Abs in these patients after receiving I-131 compared to those who did not receive I-131 as it was not randomized to determine this result. It is notable, however, that $46 \%$ of patients with antibodies at diagnosis did not have anti-Tg antibodies by the end of the study. This could be due to I-131, time from diagnosis and surgery, or be due to changes in assays over time (although the switch was to a more sensitive assay system). The disappearance in anti-Tg Abs would be useful in rendering Tg level reliable for surveillance in these patients but further studies are required to determine if RAI causes a more rapid reduction in anti-Tg Abs.

Our results support a conservative approach in low-risk patients with well-differentiated thyroid cancer that have positive anti-Tg Abs. Although not rigorously tested here, our results further suggest that an active surveillance approach to these patients using neck ultrasound and with sequential anti-Tg Abs levels may be a reasonable alternative to routine RAI administration. Indeed, the trend of anti-Tg Abs may predict the likelihood of active disease on imaging. The median time for disappearance of anti-Tg Abs is 3 years [19]. It has been reported that trend of change in anti-Tg Abs over time using the same assay system can predict the likelihood of metastatic structural disease on imaging [10, 20-22]. Therefore, the trend of anti-Tg Abs can be used to further determine the extend of further evaluation [23]. If the trend of anti-Tg Abs is concerning (i.e., rising), especially, with the absence of disease on neck ultrasound, evaluation for distant metastases on cross-sectional imaging and even empiric therapy may be justified.

Limitations of the study include that this is a retrospective study and administration of RAI was based on the treating physician's discretion, creating a possible selection bias in the population of patients who received RAI. The impact of this we believe is minimized by the strict inclusion criteria for defining low-risk disease. Another limitation is that some of the patients in group B had Hashimoto's thyroiditis (27\%) on initial pathology and that while they had no circulating anti-Tg Abs on the assays used in this study, it is possible that they were positive for anti-Tg Abs if that was evaluated using multiple anti-Tg Abs assays simultaneously as showed by Latrofa et al. [24].

In conclusion, the finding of iodine avid metastatic thyroid cancer disease in low-risk thyroid cancer patients appear to be similar in patients with positive anti-Tg Abs versus those with negative anti-Tg Abs in a carefully matched population of patients. While there was a trend in finding more iodine avid lesions in the positive anti-Tg Abs group, this was not statistically significant and was limited primarily to cervical nodal metastases that could have been potentially found with other imaging tests. The findings of this retrospective cohort study suggest that conservative management without RAI of low-risk thyroid cancer patients after total thyroidectomy may also be appropriate for low-risk patients with positive anti-Tg Abs. Further prospective studies are warranted to confirm these data.

\section{References}

1. Haugen BR, Alexander EK, Bible KC, Doherty GM, Mandel SJ, Nikiforov YE, Pacini F, Randolph GW, Sawka AM, Schlum-berger M, Schuff KG, Sherman SI, Sosa JA, Steward DL, Tuttle RM, Wartofsky L (2016)2015 American Thyroid Association management guidelines for adult patients 
with thyroid nodules and differentiated thyroid cancer: the American Thyroid Association guidelines task force on thyroid nodules and differentiated thyroid cancer. Thyroid 26(1):1-133. doi:10.1089/thy.2015.0020 [PubMed: 26462967]

2. Giovanella L, Ceriani L, Suriano S, Ghelfo A, Maffioli M (2008) Thyroglobulin measurement before rhTSH-aided 131I ablation in detecting metastases from differentiated thyroid carcinoma. Clin Endocrinol (Oxf) 69(4):659-663. doi:10.1111/j.1365-2265.2008.03244.x [PubMed: 18363882]

3. Rosario PW, Xavier AC, Calsolari MR (2011) Value of postoperative thyroglobulin and ultrasonography for the indication of ablation and (1)(3)(1)I activity in patients with thyroid cancer and low risk of recurrence. Thyroid 21(1):49-53. doi:10.1089/thy.2010.0145 [PubMed: 20954820]

4. Spencer CA, Takeuchi M, Kazarosyan M, Wang CC, Guttler RB, Singer PA, Fatemi S, LoPresti JS, Nicoloff JT (1998) Serum thyroglobulin autoantibodies: prevalence, influence on serum thyroglobulin measurement, and prognostic significance in patients with differentiated thyroid carcinoma. J Clin Endocrinol Metab 83(4):1121-1127. doi:10.1210/jcem.83.4.4683 [PubMed: 9543128]

5. Pacini F, Mariotti S, Formica N, Elisei R, Anelli S, Capotorti E, Pinchera A (1988) Thyroid autoantibodies in thyroid cancer: incidence and relationship with tumour outcome. Acta Endocrinol (Copenh) 119(3):373-380 [PubMed: 3188810]

6. Tuttle RM, Haddad RI, Ball DW, Byrd D, Dickson P, Duh QY, Ehya H, Haymart M, Hoh C, Hunt JP, Iagaru A, Kandeel F, Kopp P, Lamonica DM, Lydiatt WM, McCaffrey J, Moley JF, Parks L, Raeburn CD, Ridge JA, Ringel MD, Scheri RP, Shah JP, Sherman SI, Sturgeon C, Waguespack SG, Wang TN, Wirth LJ, Hoff-mann KG, Hughes M (2014) Thyroid carcinoma, version 2.2014. J Natl Compr Canc Netw 12(12):1671-1680 (quiz 1680) [PubMed: 25505208]

7. Lamartina L, Durante C, Filetti S, Cooper DS (2015) Low-risk differentiated thyroid cancer and radioiodine remnant ablation: a systematic review of the literature. J Clin Endocrinol Metab 100(5):1748-1761. doi:10.1210/jc.2014-3882 [PubMed: 25679996]

8. Durante C, Montesano T, Torlontano M, Attard M, Monzani F, Tumino S, Costante G, Meringolo D, Bruno R, Trulli F, Massa M, Maniglia A, D’Apollo R, Giacomelli L, Ronga G, Filetti S, Group PTCS (2013) Papillary thyroid cancer: time course of recurrences during postsurgery surveillance. J Clin Endocrinol Metab 98(2):636-642. doi:10.1210/jc.2012-3401 [PubMed: 23293334]

9. Soyluk O, Boztepe H, Aral F, Alagol F, Ozbey NC (2011) Papillary thyroid carcinoma patients assessed to be at low or intermediary risk after primary treatment are at greater risk of long term recurrence if they are thyroglobulin antibody positive or do not have distinctly low thyroglobulin at initial assessment. Thyroid 21(12):1301-1308. doi:10.1089/thy.2011.0122 [PubMed: 22136265]

10. Durante C, Tognini S, Montesano T, Orlandi F, Torlontano M, Puxeddu E, Attard M, Costante G, Tumino S, Meringolo D, Bruno R, Trulli F, Toteda M, Redler A, Ronga G, Filetti S, Monzani F, Group PTCS (2014) Clinical aggressiveness and long-term outcome in patients with papillary thyroid cancer and circulating anti-thyroglobulin autoantibodies. Thyroid 24(7):1139-1145. doi:10.1089/thy.2013.0698 [PubMed: 24702238]

11. Morbelli S, Ferrarazzo G, Pomposelli E, Pupo F, Pesce G, Calamia I, Fiz F, Clapasson A, Bauckneht M, Minuto M, Sambuceti G, Giusti M, Bagnasco M (2017) Relationship between circulating anti-thyroglobulin antibodies $(\mathrm{TgAb})$ and tumor metabolism in patients with differentiated thyroid cancer (DTC): prognostic implications. J Endocrinol Invest 40(4):417-424. doi:10.1007/s40618-016-0578-6 [PubMed: 27844413]

12. Kuo SF, Chao TC, Chang HY, Hsueh C, Lin CL, Chiang KC, Chuang WY, Chen YC, Lin JD (2015) Prognosis of papillary thyroid cancers with positive serum thyroglobulin antibody after total thyroidectomy. Asian J Surg. doi:10.1016/j.asjsur.2015.08.002

13. Jonklaas J, Cooper DS, Ain KB, Bigos T, Brierley JD, Haugen BR, Ladenson PW, Magner J, Ross DS, Skarulis MC, Steward DL, Maxon HR, Sherman SI, National Thyroid Cancer Treatment Cooperative Study G (2010) Radioiodine therapy in patients with stage I differentiated thyroid cancer. Thyroid 20(12):1423-1424. doi:10.1089/thy.2010.0308 [PubMed: 21054207]

14. Kim HJ, Kim SW (2013) Radioactive iodine ablation does not prevent recurrences in patients with papillary thyroid microcarcinoma. Clin Endocrinol (Oxf) 79(3):445. doi:10.1111/cen.12131 [PubMed: 23289927]

15. Brierley J, Tsang R, Panzarella T, Bana N (2005) Prognostic factors and the effect of treatment with radioactive iodine and external beam radiation on patients with differentiated thyroid cancer 
seen at a single institution over 40 years. Clin Endocrinol (Oxf) 63(4):418-427. doi:10.1111/ j.1365-2265.2005.02358.x [PubMed: 16181234]

16. Schvartz C, Bonnetain F, Dabakuyo S, Gauthier M, Cueff A, Fieffe S, Pochart JM, Cochet I, Crevisy E, Dalac A, Papathanassiou D, Toubeau M (2012) Impact on overall survival of radioactive iodine in low-risk differentiated thyroid cancer patients. J Clin Endocrinol Metab 97(5):1526-1535. doi:10.1210/jc.2011-2512 [PubMed: 22344193]

17. Jonklaas J, Sarlis NJ, Litofsky D, Ain KB, Bigos ST, Brierley JD, Cooper DS, Haugen BR, Ladenson PW, Magner J, Robbins J, Ross DS, Skarulis M, Maxon HR, Sherman SI (2006) Outcomes of patients with differentiated thyroid carcinoma following initial therapy. Thyroid 16(12):1229-1242. doi:10.1089/thy.2006.16.1229 [PubMed: 17199433]

18. Hay ID, Thompson GB, Grant CS, Bergstralh EJ, Dvorak CE, Gorman CA, Maurer MS, McIver B, Mullan BP, Oberg AL, Powell CC, van Heerden JA, Goellner JR (2002) Papillary thyroid carcinoma managed at the Mayo Clinic during six decades (1940-1999): temporal trends in initial therapy and long-term outcome in 2444 consecutively treated patients. World J Surg 26(8):879885. doi:10.1007/s00268-002-6612-1 [PubMed: 12016468]

19. Chiovato L, Latrofa F, Braverman LE, Pacini F, Capezzone M, Masserini L, Grasso L, Pinchera A (2003) Disappearance of humoral thyroid autoimmunity after complete removal of thyroid antigens. Ann Intern Med 139(5 Pt 1):346-351 [PubMed: 12965943]

20. Rubello D, Casara D, Girelli ME, Piccolo M, Busnardo B (1992) Clinical meaning of circulating antithyroglobulin antibodies in differentiated thyroid cancer: a prospective study. J Nucl Med 33(8):1478-1480 [PubMed: 1634938]

21. Chung JK, Park YJ, Kim TY, So Y, Kim SK, Park DJ, Lee DS, Lee MC, Cho BY (2002) Clinical significance of elevated level of serum antithyroglobulin antibody in patients with differentiated thyroid cancer after thyroid ablation. Clin Endocrinol (Oxf) 57(2):215-221 [PubMed: 12153600]

22. Kim WG, Yoon JH, Kim WB, Kim TY, Kim EY, Kim JM, Ryu JS, Gong G, Hong SJ, Shong YK (2008) Change of serum antithyroglobulin antibody levels is useful for prediction of clinical recurrence in thyroglobulin-negative patients with differentiated thyroid carcinoma. J Clin Endocrinol Metab 93(12):4683-4689. doi:10.1210/jc.2008-0962 [PubMed: 18812478]

23. Verburg FA, Luster M, Cupini C, Chiovato L, Duntas L, Elisei R, Feldt-Rasmussen U, Rimmele H, Seregni E, Smit JW, Theimer C, Giovanella L (2013) Implications of thyroglobulin antibody positivity in patients with differentiated thyroid cancer: a clinical position statement. Thyroid 23(10):1211-1225. doi:10.1089/thy.2012.0606 [PubMed: 23692026]

24. Latrofa F, Ricci D, Montanelli L, Rocchi R, Piaggi P, Sisti E, Grasso L, Basolo F, Ugolini C, Pinchera A, Vitti P (2012) Lymphocytic thyroiditis on histology correlates with serum thyroglobulin autoantibodies in patients with papillary thyroid carcinoma: impact on detection of serum thyroglobulin. J Clin Endocrinol Metab 97(7):2380-2387. doi:10.1210/jc.2011-2812 [PubMed: 22539585] 


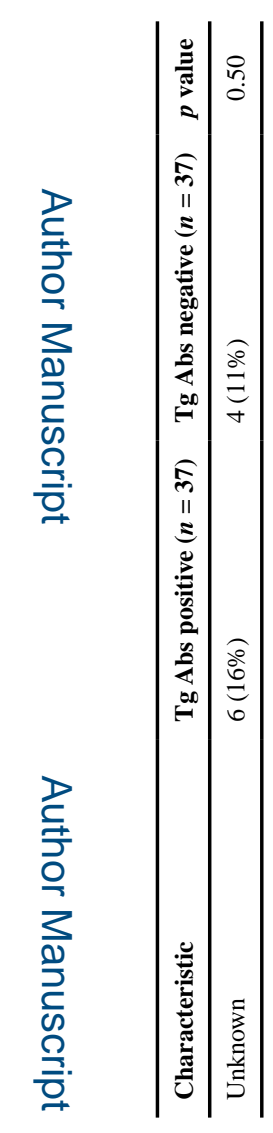

로을

를 


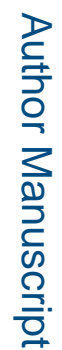

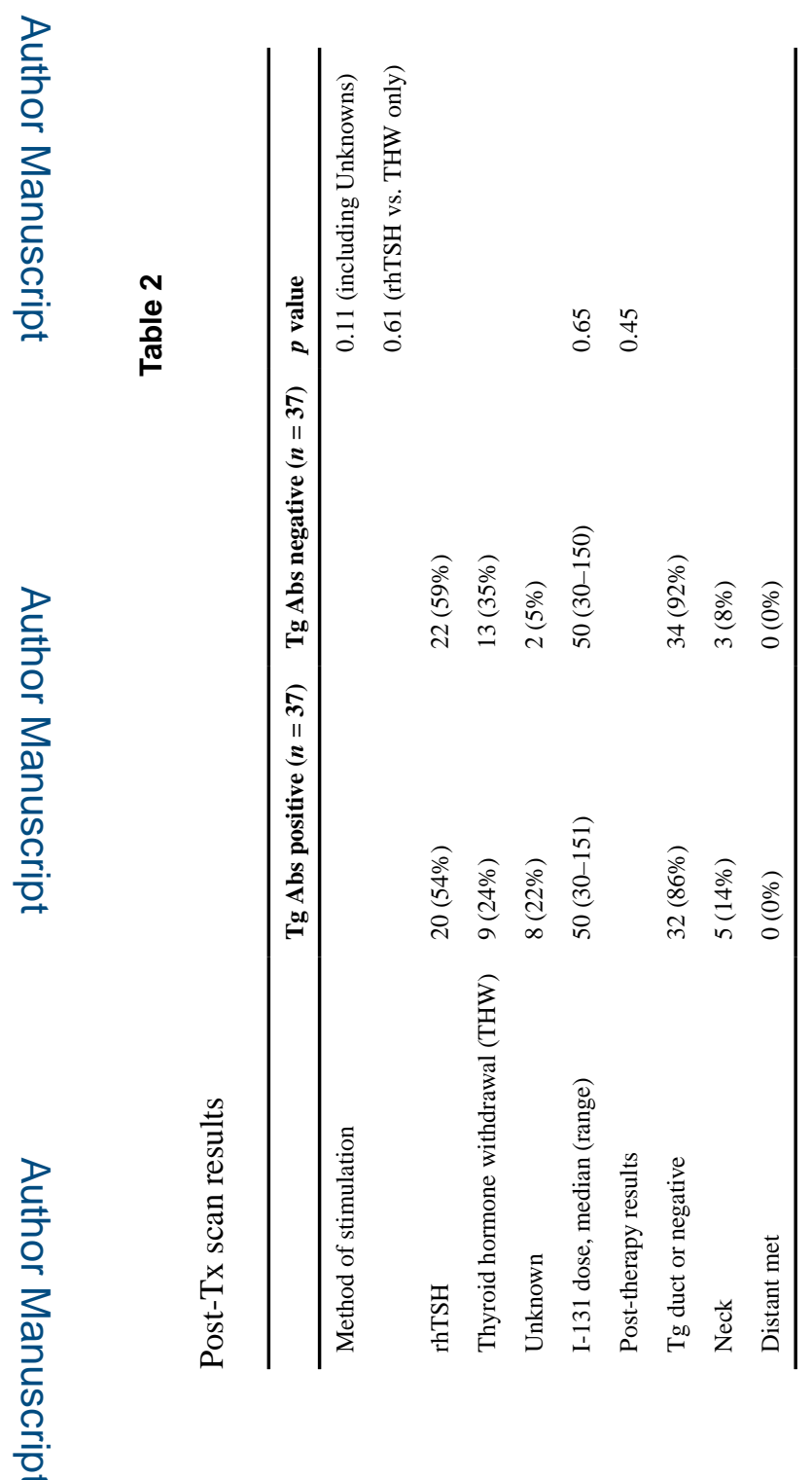

J Endocrinol Invest. Author manuscript; available in PMC 2020 March 28. 


\section{Table 3}

Follow-up and disease status at the most recent follow-up

\begin{tabular}{lll}
\hline & Tg Abs positive $(\boldsymbol{n}=\mathbf{3 7})$ & Tg Abs negative $(\boldsymbol{n}=\mathbf{3 7})$ \\
\hline $\begin{array}{l}\text { Follow-up time since RAI (months), median (range) } \\
\text { Disease status at the most recent follow-up }\end{array}$ & $37(0-118)$ & $35(3-115)$ \\
$\quad$ Free of disease & $14(38 \%)$ & \\
Indeterminate & $19(51 \%)$ & $28(76 \%)$ \\
Unknown & $4(11 \%)$ & $7(19 \%)$ \\
Biochemical incomplete & $0(0 \%)$ & $0(0 \%)$ \\
Structural & $0(0 \%)$ & $1(3 \%)$ \\
\hline
\end{tabular}

